\title{
El inmigrante en la consulta de atención primaria
} The immigrant in primary care consultations

\author{
C. Fuertes ${ }^{1,2}$, M. A. Martín Laso ${ }^{2,3}$
}

\section{RESUMEN}

En las consultas de los centros de salud, el médico de familia, el pediatra y el personal de enfermería se han encontrado, sobre todo desde finales del s. XIX y en lo que va del s. XXI, con la realidad de tener que atender a una numerosa población compuesta de personas desarraigadas de su comunidad, la mayoría de las veces sin familia cercana, con unas lenguas y culturas diferentes, con un modo distinto de entender la salud y la enfermedad. Este artículo pretende ser una aproximación al análisis de este fenómeno y contribuir a que los profesionales sanitarios puedan entender y mejorar la atención al paciente inmigrante.

Palabras clave. Atención primaria. Inmigrante. Vivencia de la salud. Patología prevalente. Competencia cultural.

An. Sist. Sanit. Navar. 2006; 29 (Supl. 1): 9-25.

\begin{abstract}
In consultations at health centres, the GP, the paediatrician and the nursing staff have faced, above all since the end of the XIX century and so far in the XXI century, the fact of having to attend to a numerous population formed of people uprooted from their community, without close relatives in the majority of cases, with different languages and cultures, and with a different way of understanding health and illness. This article analyses this phenomenon and aims to improve the understanding of health professionals and contribute to improving care for the immigrant patient.
\end{abstract}

Key words. Primary care. Immigrant. Health experience. Prevalent pathology. Cultural competence.
1. Médica de Familia de Centro Salud Chantrea. Pamplona.

2. Miembros del grupo de atención al inmigrante de la Sociedad Española de Medicina Familiar y Comunitaria (semFYC)

3. Médica de Familia de Centro de Salud Moralzarzal. Madrid.

\section{Correspondencia:}

Carmen Fuertes Goñi

Centro de Salud de Chantrea

C/ Maria Auxiliadora s/n

31015 Pamplona

Tfno. 948136272

E-mail: mfuertesg@meditex.es. 
"La medicina de la inmigración no es una medicina de parásitos ni virus raros. Es medicina de hombres y mujeres que se diferencian en la forma de expresar su sufrimiento, su modo de concebir la enfermedad, el acto médico, el dolor o la muerte. De unos hombres y mujeres que han dejado una cultura sanitaria sin haberla abandonado $y$ adquieren otra sin todavía comprenderla, de unos enfermos que esperan de nuestro mundo sanitario una tecnología carismática que los salve, pero que no aciertan a hacer comprender sus problemas interiores a médicos y cuidadores".

R. Colasanti. En: II Congreso de Medicina y Emigración. Roma, 1990

\section{VIVENCIA DE LA SALUD Y LA ENFERMEDAD DE LA POBLACIÓN INMIGRANTE}

La emigración es un fenómeno que ha tenido lugar a lo largo de toda la historia de la humanidad. Hay varios tipos de inmigrantes $^{1}$, pero en el presente artículo nos referiremos a los inmigrantes que habitualmente proceden de países en vías de desarrollo, e inician la inmigración impulsados tanto por las dificultades para llevar una vida digna en su propio país, como por las expectativas de mejorarla en el país de destino. Se mueven por tanto básicamente por motivos económicos y/o de seguridad.

El ritmo de crecimiento de la población inmigrante en España es intenso, superior al $17 \%$ anual de media, alcanzando cifras del $25 \%$ en Cataluña y superior al $20 \%$ en la Comunidad de Madrid. En Navarra la población inmigrante ha pasado de ser el $5,53 \%$ en el 2003 al $8,32 \%$ en octubre del 2005.

Con ser una realidad que se impone aceleradamente el fenómeno de la inmigración en España es mucho menos intenso que en otros países europeos con una mayor tradición de acogida y mejores condiciones económicas desde hace muchos años.

De acuerdo con Giménez ${ }^{2}$ es importante ante el fenómeno de la inmigración, ir reflexionando y favoreciendo la "integración" de los inmigrantes. Este autor define la integración como "... el proceso de adap- tación mutua de dos segmentos socioculturales mediante el cual, la minoría se incorpora a la sociedad receptora en igualdad de condiciones, derechos, obligaciones y oportunidades con los ciudadanos autóctonos, sin que ello suponga la pérdida de sus culturas de origen; y la mayoría acepta e incorpora los cambios normativos, institucionales e ideológicos necesarios para que lo anterior sea posible.".

Los centros de salud, y los servicios de urgencias son los primeros puntos de acceso al sistema sanitario y donde primero acuden los inmigrantes. Por ello para los profesionales que trabajan en estos servicios puede ser de interés conocer la vivencia de salud-enfermedad que tiene la población a la que atienden.

Para conocer la percepción de salud de los inmigrantes no basta con analizarla a partir de la población que acude a consultas sanitarias ya que en nuestro país según algunos estudios, suelen acudir a la consulta con menos frecuencia que la población española en similares circunstancias, y cuando los procesos se encuentran más avanzados $^{3}$. Esta circunstancia sin duda muy determinada por diferencias culturales en el desarrollo de los procesos de búsqueda de atención y/o por dificultades diferenciales en la accesibilidad a los servicios sanitarios públicos, nos puede llevar, como así sucede, a concluir, quizás equivocadamente, que los inmigrantes padecen problemas más severos que la población española. En una encuesta de salud del Ayuntamiento de Madrid a 8.500 personas y de ellas 1.300 extranjeros, se observó que: tienen mejor percepción de su propio estado de salud los inmigrantes de 45-65 años que los españoles de ese grupo etario, no encontrándose diferencias significativas en los otros grupos de edad; los inmigrantes tienen peor percepción de su calidad de vida en relación con la salud; no existen diferencias significativas respecto a su salud mental entre la población inmigrante y la española según los datos recogidos a través del cuestionario GHQ.

En otro estudio realizado en el Ayuntamiento de Madrid ${ }^{4}$, en los distintos grupos de discusión que se realizaron, de diferen- 
tes culturas, edad y sexo, así como en las entrevistas en profundidad, concluían que la salud es para los inmigrantes un valor esencial y básico, sobre todo porque es el vehículo necesario e imprescindible para poder trabajar.

En este estudio el concepto de salud lo articulan las diferentes culturas en relación a tres áreas.

\section{Mantenimiento del cuerpo}

En el mantenimiento del cuerpo todas las culturas dan importancia a la alimentación, aunque cada cultura hace referencia a determinados alimentos de su país de origen, bien a la calidad o la preparación de los mismos. Así los latinos echan de menos el arroz y las "arepas", los marroquíes la frescura de las frutas y verduras y los guineanos el pescado. Es importante para el profesional sanitario el conocimiento de las conductas alimentarías de los inmigrantes ${ }^{5}$, tanto para una posible intervención como herramienta para facilitar el diagnóstico o para prescribir unas determinadas conductas encaminadas a la salud del inmigrante. En el cuidado del cuerpo también incluyen la importancia de la higiene sobre todo los latinoamericanos y éstos también dan mucha importancia a la práctica del deporte y a la salud bucodental. Los marroquíes sobre todo dan importancia a una buena higiene del sueño.

\section{Representación imaginaria de la enfermedad}

En la representación imaginaria de la enfermedad hay que considerar varios aspectos. Por una parte el diferente valor de algunos síntomas o signos en algunas culturas, como la hematuria que puede ser signo de virilidad y no llevar al subsahariano a consultar por ello. La religión es especialmente importante para los musulmanes, lo que orienta la explicación de salud y enfermedad. Así entienden que se da la enfermedad como un desequilibrio entre cuerpo y alma y que puede ser efecto del incumplimiento de las normas que rigen los preceptos religiosos y hace al individuo responsable de la enfermedad. En la cultura latina el concepto de enfermedad está más ligado al cuerpo; dan mucha importancia a la exploración física, pruebas complementarias y empleo de tecnología para saber el diagnóstico de la enfermedad. Sin embargo los africanos son a veces muy reacios a que se les realice extracciones de sangre, por el vínculo que han vivido entre la sangre y diversas prácticas de brujería y magia negra.

\section{Relación que establece el inmigrante entre el cuerpo y su representación imaginaria}

La relación que establece el inmigrante entre el cuerpo y su representación imaginaria de la enfermedad, hace que siga una serie de normas o conductas. Es necesario que el profesional sanitario que atiende al inmigrante tenga en cuenta la importancia que para éste han tenido la práctica de medicinas alternativas tradicionales en su país de origen o incluso una vez asentado ya en España, puesto que puede interferir en su práctica médica habitual. Es necesario distinguir entre "curanderismo" y "remedios caseros". El "curandero" sobre todo para los latinoamericanos deja de tener importancia al venir a España, sobre todo por el fácil acceso que tiene ahora a la medicina tradicional y la práctica del curanderismo la asocian más a la falta de dinero y de cultura de su país. Los africanos son más reacios a hablar de este tema y es posible que sigan todavía creyendo en este tipo de medicina. Por otra parte el conocimiento del tipo de "remedios caseros" que ha podido emplear el inmigrante brinda una oportunidad al sanitario de establecer vínculos con los pacientes y escucharlos.

\section{FACTORES CONDICIONANTES DEL NIVEL DE SALUD DEL INMIGRANTE}

El concepto de salud es relativo y multidimensional. Cuando hablamos de salud debemos tener en cuenta elementos culturales y sociales. La Organización Mundial de la Salud define los determinantes de salud como "el conjunto de factores personales, sociales, económicos y ambientales que determinan el estado de salud de los individuos o poblaciones". Hay un vínculo bien establecido entre pobreza y bajo nivel 
de salud. Aquellos que están socialmente excluidos, como los desempleados, refugiados, inmigrantes pobres, y los sin hogar, presentan unos resultados de salud peores o mucho peores que la población general.

Los determinantes de salud de los inmigrantes se derivan de: la falta de acceso a un trabajo normalizado, de su situación irregular de residencia, del hecho de vivir en algunos casos en zonas de riesgo social elevado, de la dificultad de acceso a los servicios sanitarios en muchos casos por falta de información y de los problemas de comunicación relacionados con el idioma y las diferencias etnoculturales.

Tener en cuenta las peculiaridades diferenciales de la inmigración obliga a estar atentos a las patologías de la adaptación que tan a menudo sufre el inmigrante sobre todo por el aislamiento o la soledad; a considerar esa dura realidad por la que no siempre los inmigrantes van a disponer de los medios económicos para contar con la medicación prescrita, aunque disfruten de la tarjeta sanitaria individual; conlleva el tener cierta flexibilidad en las agendas ante las dificultades de inmigrante por horarios y la movilidad laboral; lleva al profesional sanitario a trabajar preventivamente cuando se produzcan situaciones tan habituales como la precariedad y el hacinamiento en la vivienda; y le lleva a preocuparse de los riesgos de la no contemplación de las normas de seguridad e higiene en el trabajo ${ }^{6}$.

En la tabla 1 podemos ver los principales determinantes de la salud de la población inmigrante. Destacaremos como más influyentes la vivienda y el trabajo.

Las condiciones de la vivienda del inmigrante suelen ser deficientes, tanto en salubridad como a veces, en suministro de agua y electricidad y sumado al hacinamiento favorece la transmisión de algunas enfermedades infecciosas (tuberculosis, diarreas, sarna etc.) así como los problemas derivados de la falta de espacio y de privacidad. El trabajo del inmigrante con frecuencia es en empleos temporales y en malas condiciones, desconociendo las maquinarias y las medidas básicas de prevención de accidentes lo que favorece los accidentes laborales. Estos accidentes los padecen tres veces más que la población adulta española ${ }^{7}$. El problema se complica si el inmigrante está en situación de irregularidad o si desconoce el idioma. Uno de los sectores principales de actividad laboral de los inmigrantes es el agropecuario donde el riesgo de exposición a pesticidas y otros productos químicos es un problema frecuente y en España se ha asociado a depresión, trastornos neurológicos y abortos en trabajadores agrícolas inmigrantes. Otros problemas de salud frecuentes son la deshidratación y algunos padecimientos cardíacos en relación con las altas temperaturas que soportan habitualmente los trabajadores de los invernaderos ${ }^{8}$. Otros sectores de trabajo del inmigrante son la construcción (15\%) y hostelería (12\%). Las mujeres inmigrantes sobre todo trabajan como empleadas de hogar con la consiguiente soledad y aislamiento $\mathrm{y}$ el tener que asumir normas y pautas de conducta que frecuentemente no son las suyas ${ }^{9}$.

\section{PATOLOGÍA PREVALENTE DEL INMIGRANTE EN LA CONSULTA DE ATENCIÓN PRIMARIA}

Los inmigrantes en general no llegan enfermos a España, su vulnerabilidad empieza en el país receptor, por los hábitos y situaciones sociales adquiridas, las malas condiciones laborales y de vivienda y los pocos apoyos familiares y sociales, con las consiguientes repercusiones psicológicas $^{10}$.

El inmigrante llega a nuestro país con un importante capital de salud, son personas generalmente sanas y jóvenes que no suelen representar ningún riesgo para la salud de la comunidad receptora. Como consecuencia del mayor tiempo de estancia en nuestro país y del mayor número de hijos que tienen los inmigrantes, las patologías atendidas tienen cada vez más relación con el estilo de vida occidental y con las condiciones laborales y familiares. Así cada vez es más frecuente la inclusión de inmigrantes en los programas de crónicos del centro de salud como en el de hipertensión o diabetes. Las enfermedades que encontramos en los inmigrantes podemos agruparlas en 4 categorías observando su 
Tabla 1. Determinantes de la salud de la población inmigrante.

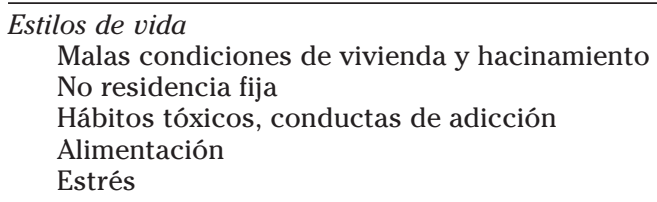

La mujer inmigrante en algunas culturas está supeditada al hombre y tiene más dificultad de integración en el país receptor

\section{Cultura sanitaria}

Desconocimiento del funcionamiento de los servicios sanitarios

Diferente concepto salud/enfermedad

Prácticas de medicina tradicional

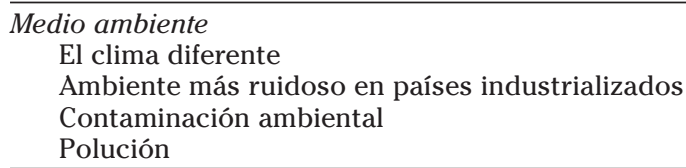

distinta importancia según se representa en la pirámide que podemos ver en la figura 1.

\section{Enfermedades importadas}

Las enfermedades importadas (malaria, esquistosomiasis, enfermedad de Chagas...) son el grupo menos numeroso y tienen lugar especialmente en hijos y en inmigrantes de larga estancia en nuestro país, que han perdido parte de su inmunidad natural y retornan de forma transitoria a sus países de origen y en los hijos de inmigrantes que han nacido en España que viajan al país de sus padres sin inmunidad y sin profilaxis. El riesgo de transmitir una enfermedad importada a la sociedad de acogida es mínimo ${ }^{11,12}$, ya que no se dan las circunstancias ecológicas ni los vectores necesarios para su trasmisión (clima, saneamiento ambiental, falta de huéspedes intermediarios, etc.). En los inmigrantes el riesgo de padecer enfermedades importadas disminuye con el tiempo de estancia en el país de destino, salvo algunas enfermedades que pueden manifestarse muchos años después de abandonar la zona endémica, como son: lepra tubercu- 


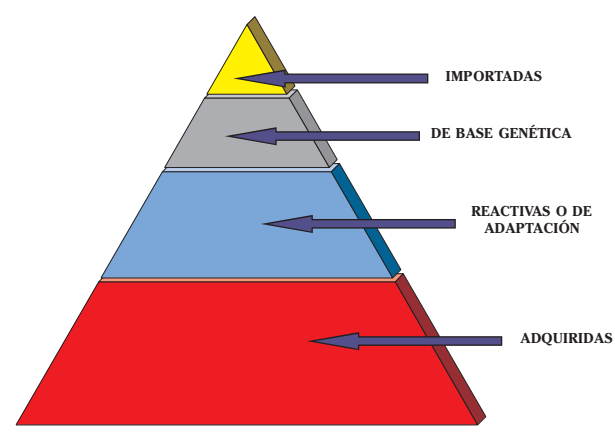

Figura 1. Enfermedades de los inmigrantes.

losis, sífilis, VIH, esquistosomiasis, la infestación por Strongyloides stercolaris que puede permanecer hasta 40 años latente y se manifiesta cuando el paciente se inmunodeprime, enfermedad de Chagas, toxoplasmosis, y hepatitis virales ${ }^{13}$. La malaria se debe sospechar en atención primaria ante todo inmigrante que lleve poco tiempo en nuestro país, o que acabe de regresar de un viaje de su país sin que hubiera hecho quimioprofilaxis, y que presente fiebre persistente sin foco. Especialmente grave es en el caso de niños entre 1 y 5 años que frecuentemente suelen desencadenar un síndrome nefrótico; y también es grave en mujeres embarazadas por el riesgo para el feto. Hasta ahora los casos de malaria declarados en España son casos importados, fundamentalmente debido a viajeros y la especie suele ser $P$. falciparum. En el 2002 se habían declarado en España $452 \operatorname{casos}^{14}$, aunque se estima que pudo haber unos 600 casos, de éstos, el $60 \%$ están provocados por viajes a lugares de riesgo y el $40 \%$ corresponden a inmigrantes. La confirmación del diagnóstico se hace con el análisis de gota gruesa. Las personas procedentes de países endémicos pueden estar infectadas y no manifestar ninguna sintomatología a causa de la exposición constante al plasmodio en su país, y presentar la enfermedad una vez transcurridos tres meses de su llegada, o bien pueden sufrir crisis palúdicas leves o tener el protozoo en sangre y estar asintomáticos ${ }^{15}$.

Entre las enfermedades infecciosas transmisibles o cosmopolitas pueden con- siderarse: la tuberculosis, hepatitis virales, infecciones de transmisión sexual incluyendo el sida, y muchas parasitosis. De entre las infecciosas merece una mención especial la tuberculosis, que está ligada no tanto a la situación de inmigración, sino a la mayor prevalencia de tuberculosis en el país de origen y al hacinamiento y pobreza en que vive el inmigrante en el país receptor y que favorece la transmisión de la enfermedad. La atención al inmigrante con tuberculosis conlleva dificultades adicionales a las propias de la atención a una enfermedad que requiere un tratamiento largo y que es considerada vergonzante por casi todas las poblaciones. Además es importante recordar que algunos síntomas clásicos, como la hemoptisis o la sudoración nocturna pueden ser difíciles de que el inmigrante los comente por barreras culturales o idiomáticas. A esto se añade la dificultad de hacer quimioprofilaxis a todos los contactos del inmigrante con tuberculosis y en ello el médico de familia tiene un papel fundamental. En cuanto al tratamiento generalmente los inmigrantes requerirán el tratamiento con 4 fármacos dadas las altas tasas de resistencia a la isoniacida en esta población que es del 7$10,5 \%{ }^{16}$. Lo más difícil es conseguir que se complete el tratamiento y también hay que tener en cuenta la posibilidad de una mayor incidencia de hepatotoxicidad en una población con elevada prevalencia de portadores crónicos de hepatitis B.

Hay distintos estudios epidemiológicos para determinar los factores que influyen en la incidencia de tuberculosis entre los inmigrantes y entre la población autóctona de distintos países, y en algunos de estos estudios $^{17-19}$, se han aplicado técnicas de epidemiología molecular tanto en los de países europeos como de EEUU y se ha concluido que:

a) ninguno de los inmigrantes estudiados se había infectado recientemente en la ciudad donde habitaba. La hipótesis más probable es que se hayan infectado en su país de nacimiento y que se produzca la progresión a enfermedad después de la llegada debido a las condiciones insalubres de vivienda y nutrición y a condiciones laborales precarias; 
b) no se demostró la trasmisión desde la población inmigrante a la población autóctona;

c) los factores asociados en la población autóctona fueron VIH positivo, abuso de drogas por vía parenteral, indigencia, sexo masculino, edad inferior a 35 años y antecedente de estancia en prisión.

Hay diversos estudios de incidencia, prevalencia y contactos en inmigrante ${ }^{20-23}$, encontrando que los datos varían mucho de unos a otros, así la prevalencia de tuberculosis en los asentamientos de Ceuta ${ }^{24}$ que acogen a inmigrantes recién llegados es de $32,5 \%$, similar a algunos sectores de Barcelona y Madrid $^{4,25}$. En la provincia de Madrid la proporción de casos de tuberculosis en extranjeros pasó de 3,2 en 1994 a 14,9\% en el año 2000 y en este mismo año la prevalencia de tuberculosis para el conjunto de la población inmigrante ${ }^{26}$ era del $2,5 \%$.

\section{Patologías de base genética}

Las patologías de base genética representan un número pequeño dentro del global de patologías del inmigrante atendidas en atención primaria. Sólo destacar la mayor prevalencia en relación a la población autóctona de algunas anemias hemolíticas como el déficit de G6PDH o algunas hemoglobinopatías.

\section{Patologías reactivas o de adaptación y las adquiridas en nuestro medio}

Las patologías reactivas o de adaptación y las adquiridas en nuestro medio ${ }^{27}$ son por último y como vemos en la base de la pirámide de la figura 1, las patologías del inmigrante que más frecuentemente encontramos en atención primaria. Las patologías reactivas o de adaptación se expresan fundamentalmente con somatizaciones y síntomas vagos que indican una problemática relacional, de desarraigo, de falta de integración, de problemas de convivencia o de falta de recursos ${ }^{28}$, es decir son enfermedades psicosomáticas. Este grupo de enfermedades están condiciona- das por múltiples factores de riesgo como puede apreciarse en la figura 2.

Los problemas por las que consulta el paciente inmigrante en el centro de salud ${ }^{29-31}$, son los mismos que las de la población autóctona ${ }^{32}$ : problemas de infecciones respiratorias agudas (20\%), osteomusculares (19\%), cefalea (12\%), de salud sexual y reproductiva $(10 \%)$, digestivos $(9 \%)$, accidentes (5\%), y otros (incluyendo problemas de piel y síntomas y signos mal definidos (26\%). En la figura 3 puede apreciarse la distribución de motivos de consulta de los inmigrantes en un estudio realizado en Centro de Salud de Vícar (Almería) y puede observarse que un porcentaje importante (16\%) de la población inmigrante no acudió a la cita concertada; quizás por dificultades en relación a ausentarse del puesto de trabajo, o por haber mejorado de los síntomas que presentaba al solicitar la cita. En otro $23 \%$ no se determinó el motivo de consulta porque probablemente serían del grupo de síntomas y signos mal definidos. Estas consultas suelen ser el reflejo de problemas psicosomáticos así como también los reflejan las consultas por: impotencia, trastornos del sueño, mialgias repetidas, dolores abdominales o torácicos inespecíficos, cefaleas, etc. $^{33}$. Estos síntomas suelen ser la expresión de un sufrimiento psicológico muchas veces no reconocido ${ }^{34}$.

\section{EL DUELO MIGRATORIO Y EL ABORDAJE DE LA SALUD MENTAL DE LOS INMIGRANTES EN LA CONSULTA DE ATENCIÓN PRIMARIA}

El paciente inmigrante tiene que adaptarse a muchas situaciones diferentes a las que vivía en su país de origen, por lo que es más probable que presenten mayores tasas de depresión y trastornos de ansiedad que grupos con idénticas características sociodemográficas de su país de origen o de la cultura anfitriona ${ }^{35}$. En algunos casos se añade a las dificultades anteriores, las condiciones del desplazamiento desde su país de origen, y otros acontecimientos que le han podido suceder al inmigrante, así como dificultades ambientales ligadas a la inmigración, todo lo cual hace que el trastorno por estrés postraumático sea más prevalente entre el grupo de 


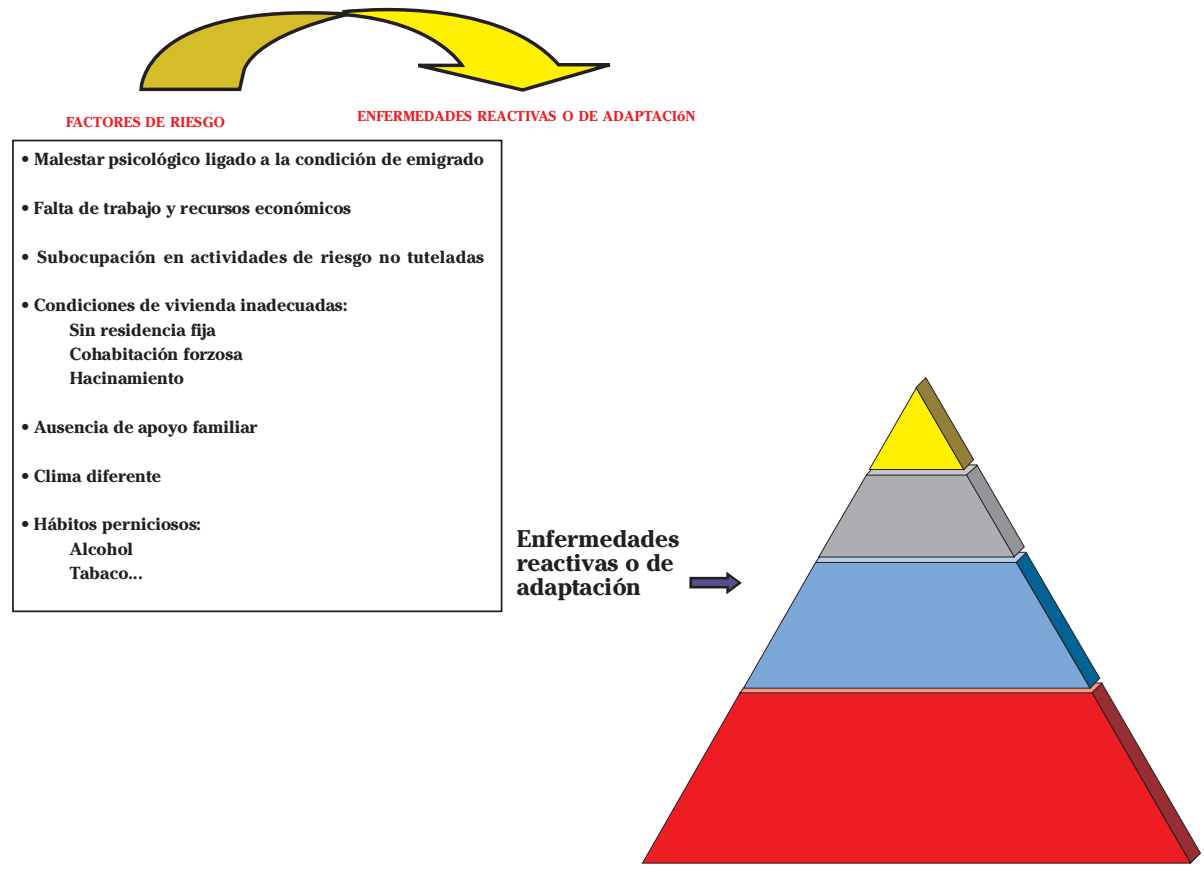

Figura 2. Factores de riesgo en las enfermedades reactivas o de adaptación.

pacientes inmigrantes, sobre todo en aquellos que han sufrido represiones y en los refugiados políticos.

La mayoría de la patología de salud mental, que observamos en las consultas de atención primaria, en la atención al paciente inmigrante son trastornos de adaptación, somatizaciones y los relacionados con el duelo migratorio.

El inmigrante es preciso que elabore un duelo, porque ha dejado una parte importante de su vida en el país de origen. Podemos diferenciar dos tipos de duelo migratorio: el simple, que se produce cuando el sujeto emigra en buenas condiciones personales y el medio con el que se encuentra le permite desarrollar con éxito el proyecto migratorio, y el duelo complicado en el que por múltiples circunstancias hay dificultades en la elaboración del duelo migratorio y pueden aparecer alteraciones en el equilibrio psíquico del sujeto.

Achotegui $^{36}$ señala que habría al menos 7 duelos en la migración y su enumeración y las características del duelo se pueden apreciar en la tabla 2. Llamamos proceso de duelo a la forma que tienen las personas de hacer frente a las pérdidas. Las formas externas en las que el duelo se manifiesta están canalizadas por la cultura y por eso es decisivo conocer cómo marca la

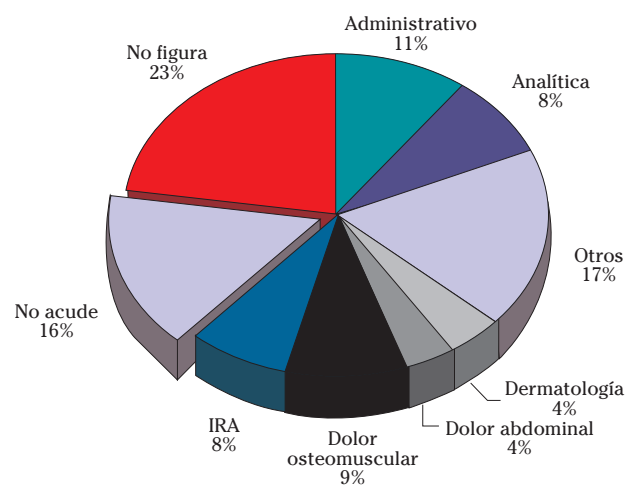

N: 1.343 (7\% de todas las consultas).

Figura 3. Motivos de consulta de los inmigrantes en el centro de Salud de Dicar (Almería). 
Tabla 2. Características del duelo en el paciente inmigrante.

\begin{tabular}{lll}
\hline Duelo múltiple y de aspectos arraigados & Duelo por: & 5) El status social \\
en su personalidad & 1) La familia y amigos & 6) Contacto con la etnia \\
& 2) La lengua & 7) La vivienda, trabajo \\
\hline Parcial & 3) La cultura & 4) La tierra \\
& El objeto del duelo, es decir lo relativo al país de origen, \\
& no desaparece. & Es el duelo de una separación más que de una pérdida. \\
\hline Recurrente & El ir y venir al país de origen y la relación con los \\
& contactos, hacen que los recuerdos se reactiven, que \\
& sea un duelo de larga duración y en fases recurrentes. \\
\hline Ambivalente & Se han perdido elementos del país de origen pero el \\
\hline Transgeneracional & país de acogida le brinda otras oportunidades. \\
\hline
\end{tabular}

cultura de origen del inmigrante que se haga el duelo. Expresiones emocionales que al médico le resultan extrañas pueden ser manifestaciones del duelo que el paciente está haciendo, realizadas desde su idiosincrasia cultural.

Mencionaremos también el denominado síndrome del inmigrante con estrés crónico y múltiple (Síndrome de Ulises) descrito por Achotegui ${ }^{36}$ y que se caracteriza porque el inmigrante padece determinados estresores o duelos y por otro lado porque aparecen una serie de síntomas psiquiátricos relacionados sobre todo con la soledad y separación forzada de los seres queridos, con el sentimiento de desesperanza y fracaso que surge cuando el inmigrante no logra ni siquiera las mínimas condiciones para salir adelante y con los peligros físicos relacionados con el viaje migratorio. Este estrés se caracteriza por ser crónico y múltiple. Es un estrés que el inmigrante no puede controlar y que se vive con muy poco apoyo social. Esta patología, el médico de familia la ha de sospechar y derivar al paciente al especialista de salud mental si lo considera oportuno. También en algunas comunidades autónomas como Navarra, en algunos centros de salud, en colaboración con Cruz Roja, se ha considerado el abordaje del síndrome de Ulises desde una perspectiva de prevención y terapia grupal con inmigrantes que ya llevan un tiempo en España.

\section{LA COMUNICACIÓN CON EL PACIENTE INMIGRANTE COMO UN RETO: COMPETENCIA CULTURAL Y ENTREVISTA CLÍNICA}

La atención sanitaria a personas inmigradas, salvo sus peculiaridades culturales, no difiere significativamente de la que se presta a personas autóctonas; si bien en los primeros meses de su llegada y durante un periodo limitado de tiempo requieren una atención más específica.

Un principio ético de la asistencia sanitaria, el principio de justicia de la bioética moderna $^{37,38}$ habla de la obligación de tener en cuenta a todo el mundo con la misma consideración y respeto en el orden social, y a no discriminar a nadie por razón de etnia, sexo, creencias, edad, etc., promoviendo así la igualdad de oportunidades. Sin embargo, tratar a todo el mundo en condiciones de igualdad no significa tratar a todo el mundo igual. El profesional sanitario debe saber atender a la población, considerando los aspectos diferenciales de cada uno, de manera que pueda personalizar la asistencia teniendo en cuenta la diversidad y las diferencias culturales y religiosas que influyen en la forma de entender la salud y la vida en general ${ }^{39}$. El profesional sanitario vive con cierto estrés y ansiedad la atención al inmigrante como consecuencia fundamentalmente de las propias características actuales del sistema (falta de tiempo, exceso de demanda, 
desequilibrios en el número de tarjetas sanitarias adscritas a cada profesional, restricciones al acceso a determinadas pruebas complementarias, etc..); unidas a las características específicas de este colectivo (idioma, dudas sobre interpretación correcta de síntomas y signos, incertidumbre ante patologías poco conocidas, medios diagnósticos de laboratorio inadecuados, problemas de adherencia a los tratamientos y seguimiento, etc.).

Los problemas con los que se enfrenta el profesional sanitario en las consultas de los centros de salud en cuanto a la atención al paciente inmigrante son sobre todo problemas lingüísticos, de interferencias culturales y de interferencias emocionales.

El profesional sanitario ha de educarse en competencia cultural. Al hablar de salud debe tener en cuenta elementos culturales y sociales. Cuando atiende a inmigrantes, el objetivo debe ser una atención médica culturalmente apropiada, eficiencia clínica culturalmente competente, respetuosa, flexible y sensible. Debe tener presente que la concepción de la salud y de la enfermedad puede ser distinta de la suya. Martincano define la medicina transcultural $^{40}$ como "los conocimientos médicos y de comunicación aplicados entre un sanitario de un determinado grupo étnico y un paciente de otro", abarca tanto aspectos físicos, psicológicos y sociales como aspectos de la cultura, religión o etnia. En el "Manual de medicina transcultural" ${ }^{40}$ se recogen muchas recomendaciones para minimizar la disonancia cultural con los pacientes extranjeros y como dice el autor "si se está dispuesto, sensible y culturalmente, nos bastarán unos pocos conocimientos referidos a la interculturalidad para que aparezca la sintonía y se dé lugar a la mutua confianza y a la aceptación de nuestros eficientes cuidados clínicos".

Hay muchos modelos de medicina transcultural ${ }^{40}$. Citaremos algunos de los más importantes:

- Modelo del amanecer: el cuidado de la salud tiene semejanzas y diferencias de acuerdo con el contexto cultural en que se encuentra. Es el modelo más popular. Es un modelo sobre todo práctico para la enfermería transcultural, ya que la enfermera debe identificar, en los sujetos a quien cuida, aquellas prácticas y valores dignos de mantener, porque promueven el bienestar y condiciones de vida adecuadas para la conservación de la salud.

- El modelo del perfil del material cultural de Murdock, tiene como referencia la SCCS que son bases de datos de variables sobre poblaciones culturalmente diferentes, creadas en 1971.

- El Health Care Industry: estudio de los fenómenos culturales y las estrategias de ajuste a una escena cultural diferente.

- Sistema CARE, proceso de Competencia Cultural en la Oferta de los servicios de Salud y preguntas ASKED: promueve la competencia cultural.

- Teoría de Purnell para la Competencia Cultural (1998): sistema de trabajo comprensivo, sistemático y conciso para enfrentarse a la diversidad cultural.

La competencia en medicina transcultural es fundamental para que el profesional sanitario realice una buena entrevista clínica. Las peculiaridades del paciente inmigrante debemos tenerlas en cuenta en toda entrevista clínica. En ésta un factor importante para tener un buen resultado es el cómo se realiza la acogida. En el caso de paciente inmigrante además de la cordialidad que vehicularemos sobre todo a través de la comunicación no verbal, debemos realizar algunas preguntas que nos ayuden a situarnos en la realidad del paciente que tenemos delante. Estas preguntas son con relación a: ¿quién es el paciente? ¿de dónde procede?, ¿cómo era su situación en su país de origen? ¿por qué ha emigrado? ¿en qué condiciones? ¿qué situación se ha encontrado aquí?

Deberemos adquirir habilidades necesarias para realizar una buena entrevista clínica ${ }^{41}$ y para ello hay que tener en cuenta y llevar a la práctica algunos elementos importantes de ésta como son: la calidez y cordialidad, la empatía, el respeto, la asertividad y la baja reactividad. Es clave prestar una escucha activa a los problemas del paciente y desarrollar habilidades para 
ello. Si el paciente no habla nuestro idioma hay que prestar especial atención a la comunicación no verbal y a la exploración e intentar buscar un mediador cultural cualificado que pueda hacernos de traductor $^{41}$. Hay varios modelos de tareas propuestas para la relación clínica ${ }^{42}$. En el caso del paciente inmigrante quizás el modelo mejor para llevar a cabo una buena entrevista clínica se base en el modelo de comunicación centrada en el paciente de Wenstern Ontario ${ }^{43}$. Este modelo define las siguientes tareas: 1) explorar la enfermedad y la dolencia; 2) comprender a la persona; 3) buscar puntos comunes respecto al plan; 4) incorporar la prevención y promoción de la salud; 5) incrementar la relación médico-paciente; 6) ser realista.

En la comunicación profesional sanitario-paciente, ante el fenómeno de la inmigración surge para el profesional sanitario un nuevo reto. En un principio, en los años 80 , cuando la afluencia de inmigrantes no era tan masiva se consideraba más o menos que el inmigrante, generalmente magrebí, tenía una estancia temporal en España, sobre todo en Andalucía y Levante (magrebís jóvenes que venían para labores temporales de agricultura). La comunicación se solventaba con la pericia del profesional y la esperanza de que la temporada acabara y el paciente no volviera a apare$\operatorname{cer}^{44}$. Sin embargo, esto ha cambiado y fundamentalmente se crean en cuanto a la comunicación médico-paciente, problemas con relación a tres aspectos.

\section{Problemas generados por el sistema}

Al comienzo del fenómeno migratorio en España, el principio de "universalidad de la asistencia" no estaba garantizado totalmente para los "sin papeles" ni en todas las situaciones. Por otra parte, encontraba un sistema sanitario muy burocratizado y desconocido para el inmigrante con dificultades idiomáticas, siendo la figura del traductor a veces muy necesaria. En muchos casos el sistema sanitario es muy diferente al de su país de origen y acaba acudiendo a urgencias a veces por problemas que se pueden atender en atención ordinaria en el centro de salud.

\section{Problemas con relación al profesional sanitario}

La dificultad idiomática de nuevo crea angustia en el profesional que tiene que recurrir a primar la comunicación no verbal y la exploración física. Además cuenta con la limitación del tiempo por consulta, que es muy poco, y a pesar de que sería necesario en el caso de la consulta del inmigrante disponer de más tiempo, no lo tiene, y tiene que asumir esto como una limitación.

Aunque no son las más frecuentes el médico de familia ha de pensar y repasar las enfermedades importadas o las de base genética y ello le genera ansiedad.

Las diferencias culturales y religiosas entre la población inmigrante son importantes, ya que el concepto salud y enfermedad es diferente en las distintas culturas, pueden presentar enfermedades similares a la población autóctona pero tener para ellos diferente significado, así la infertilidad en la mujer africana es un drama para ella más importante que para una mujer española el padecer cualquier enfermedad incurable. También hay que tener en cuenta síntomas que son reflejo de enfermedades importantes pero que el inmigrante no va a consultar por ellos por no darles importancia ya que son habituales en su medio v.g. la fiebre que periódicamente tienen al padecer un paludismo crónico. Existe el peligro de que el médico de familia banalice las quejas psíquicas y "achacarlo" todo al desarraigo. Es importante que el profesional sanitario aborde la idea que tiene el paciente sobre la naturaleza de la enfermedad, los sentimientos y miedos que le produce, el impacto en su vida diaria, las expectativas que tiene con respecto al profesional. Tener en cuenta a la hora de prescribir una dieta o algunos fármacos, el tipo de alimentación de su país y las creencias religiosas v.g. es importante en los musulmanes saber si están en el período del ramadán e intentar dar medicaciones de dosificación única que puedan tomarla a la noche.

El profesional sanitario ha de tener en cuenta alguna de las características comunes en la población inmigrante, la movilidad geográfica con frecuentes cambios de 
domicilio y no ha de dejarse llevar por los sentimientos de incomodidad o paternalismo que a veces todos estos factores le generan de forma inconsciente.

\section{Problemas con relación al paciente inmigrante}

Los emigrantes en general son personas sanas y fuertes, pero esto no significa que después de un largo viaje a veces en precario y unido a situación de marginación, hacinamiento y pobreza, no se favorezca que hayan podido desarrollar alguna enfermedad. Por otra parte llegan a un medio desconocido y tienen sus miedos, inseguridades, muchos están "irregulares". Las dificultades idiomáticas para el inmigrante también le suponen un problema y a veces acuden a las consultas utilizando a sus hijos como traductores, puesto que son los niños los que aprenden más rápidamente el idioma.

En unos casos desconfían de la medicina occidental pero en otros pueden pensar en ella como algo milagroso. En algunos contextos como en el mundo árabe se plantea un problema si la atención sanitaria a un varón se la ha de prestar una mujer o viceversa.

El profesional sanitario de atención primaria ha de desarrollar habilidades para poder solventar estos problemas y ser competente culturalmente para atender al paciente inmigrante desde atención primaria. Las sociedades científicas y consejerías de salud tienen un papel de contribuir a la formación y ayudar a ello promoviendo guías o recomendaciones que faciliten esta atención ${ }^{45-47}$.

\section{ATENCIÓN A LA FAMILIA, AL NIÑO Y A LA MUJER INMIGRANTE}

En atención primaria de salud es importante conocer las características de las familias inmigrantes y los conflictos que presentan para poder intervenir desde una perspectiva de atención familiar.

Algunas mujeres inmigrantes están sujetas a una carga extra de violencia social y psicológica ya que a la situación desfavorable de inmigrante que comparten con los hombres, se suma la de género.
La consideración social de la mujer inmigrante está influida por la cultura de la sociedad de la cual procede.

En cuanto al embarazo y lactancia, la lactancia materna es una pauta bien valorada en general por todas las culturas de inmigrantes, aunque se debe recordar que es necesaria la introducción de alimentación complementaria en el niño a partir del $5^{\circ}-6^{\circ}$ mes de vida, ya que en una lactancia materna prolongada, no suplementada, puede producir anemia ferropénica. Por otra parte en las mujeres inmigrantes son más frecuentes los partos prematuros, en las africanas casi el doble que entre las españolas y los niños de aquéllas tienen bajo peso al nacer en un 11,5\% comparado con el 5,5\% en las españolas ${ }^{48}$.

La morbimortalidad neonatal observada tiene más relación con los datos relativos al adecuado control y seguimiento del embarazo y las condiciones sociosanitarias en las que vive la mujer inmigrante. Estos condicionantes también influyen en la morbilidad de la mujer inmigrante $y$ podemos resumirlos en los siguientes:

- El trabajo de la mujer inmigrante está limitado la mayoría de las veces al sector del servicio doméstico en el que sufren mayor aislamiento, al ser más difícil relacionarse con sus redes sociales nativas en el país de acogida sobre todo las mujeres que trabajan como empleadas de hogar internas.

- Hay mujeres que siguen viviendo en el seno de familias tradicionales que exigen de ellas determinados comportamientos clásicos, distintos de lo que se espera de ellas en los trabajos que realizan, provocando interferencias entre una cultura y otra. Todo esto se traduce en síntomas muy vagos por los que acuden a la consulta de Atención Primaria: astenia, cefaleas, lumbalgias, cervicalgias, tristeza, insomnio, etc. o específicamente alteraciones psicológicas.

- Otras mujeres se ven abocadas a tener que ejercer la prostitución: por la falta de capacitación, la indocumentación y la necesidad de dinero. Ello les hace estar más expuestas a enfermedades de transmisión sexual, violencia sexual 
y embarazos no deseados.

Con relación al niño inmigrante, según diversos estudios, los problemas relacionados con la nutrición son frecuentes. La utilización inapropiada de los sustitutos de leche materna y la baja ganancia ponderal se han relacionado con deficiencias vitamínicas y anemia ferropénica en hijos de inmigrantes en España y la alimentación es deficitaria en algunos componentes básicos de la dieta. Muchos de estos niños pasan gran parte de día solos y esto provoca alteraciones en las relaciones afectivas. Así mismo se ha detectado falta de conocimientos básicos de puericultura por parte de las madres y problemas de seguimiento de las pautas dietéticas recomendadas y de los tratamientos prescritos. La constante movilidad en ocasiones dificulta el correcto seguimiento y las revisiones de los niños en las consultas de atención primaria.

Si la atención primaria tiene entre sus funciones la tarea importante de la prevención de salud, esta lo es más todavía con la población inmigrante y son tareas prioritarias las actividades preventivas como son la actualización y aplicación de un correcto calendario vacunal y las del programa materno-infantil.

Por otra parte en los niños, y también en los adultos, es importante repasar el calendario vacunal y las medidas preventi- vas, ya que se han detectado algunos problemas en este grupo como: escasa cobertura vacunal en países de origen, disminución de la eficacia de las vacunas por problemas de conservación, ausencia de documentos justificativos de vacunaciones previas, mayor incidencia de casos de enfermedades transmisibles, viajes a países de origen y no observar las debidas precauciones.

\section{ACTUACIONES PARA LA ATENCIÓN AL PACIENTE INMIGRANTE Y PROPUESTAS DE MEJORA}

Las actuaciones que habría que llevar a cabo en atención primaria con el paciente inmigrante vienen resumidas en la tabla 3 . Muchas de ellas son del ámbito de la atención comunitaria de salud y en este sentido es necesario un trabajo interdisciplinar del médico de familia, la enfermera, la trabajadora social y los distintos organismos del barrio o la comunidad en que ejerce su atención el centro de salud.

Es importante incorporar modelos de salud comunitaria que permitan la participación de mediadores culturales de salud, figura puente entre los servicios de atención primaria y las comunidades inmigrantes, que favorezcan una atención adecuada.

Hace falta llevar a cabo los estudios precisos sobre necesidades de salud de la

Tabla 3. Actuaciones en atención primaria ante el paciente inmigrante.

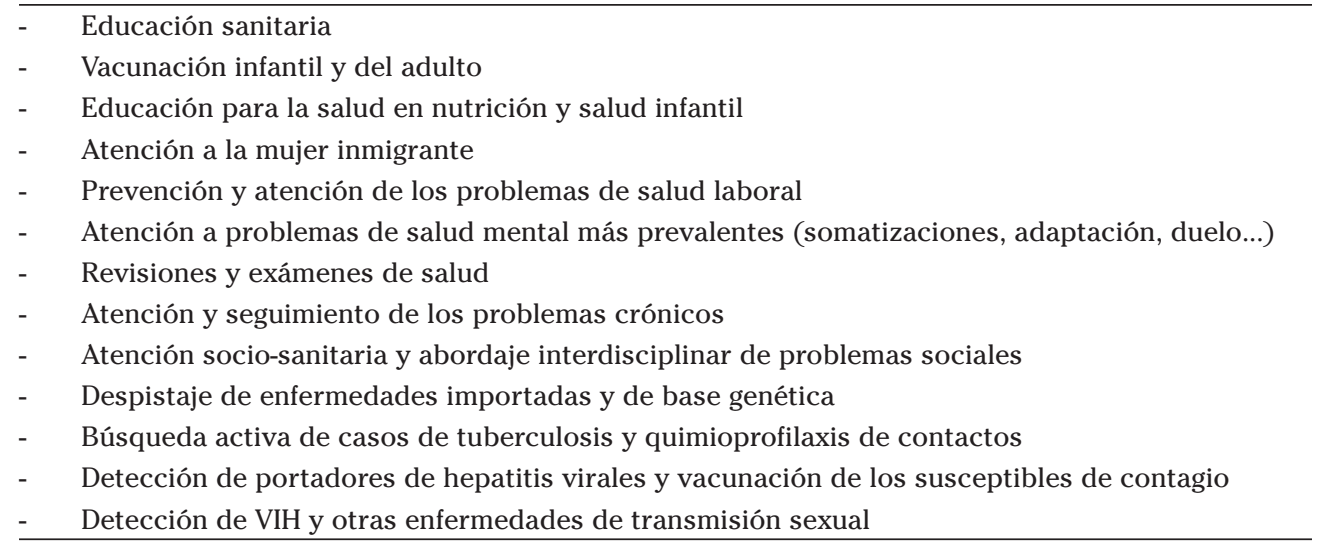


población inmigrante, mejorando los registros e incorporando las variables adecuadas que identifiquen estas nuevas poblaciones.

Por otra parte se deben diseñar algunas estrategias ${ }^{33}$ para lograr una mejor atención sanitaria al paciente inmigrante tanto desde la Administración como de los profesionales que trabajan en ellas ${ }^{49,50}$. Algunas propuestas pueden ser:

a. Formación de profesionales sanitarios y no sanitarios, con el objeto de fomentar la formación de aspectos relacionados con la inmigración (comunicación intercultural, enfermedades importadas, respeto a los valores de otras culturas...) Los profesionales sanitarios deben trabajar para desarrollar competencias asistenciales que tengan en cuenta la diversidad cultural y los elementos de esa competencia deberían figurar en los programas de formación tanto de pregrado como postgrado. Las autoridades sanitarias, por otro lado, deben proporcionar formación continuada y exigir unos mínimos de competencia cultural en la acreditación de centros y servicios para así poder afrontar mejor los retos de la diversidad, favorecer el acceso a los servicios de las personas de otras culturas y reducir la discriminación en el ámbito de la salud y la asistencia sanitaria.

b. Favorecer la formación de asociaciones de inmigrantes, con el fin de ofrecer información que facilite el acceso a una atención sanitaria adecuada y desarrollar programas de promoción de la salud y educación sanitaria. Se puede ofertar cursos y programas o grupos de ayuda.

c. Potenciar la figura del mediador cultural, que en otros países ha demostrado ser un instrumento muy eficaz para facilitar el trabajo de puente entre distintas comunidades. Su perfil es el de una persona miembro del colectivo con el que ha de mediar. Conoce la cultura y el idioma de su país y los del de acogida, es respetado en su comunidad y tiene capacidad de negociación.

d. Elaboración de guías y protocolos de atención al paciente inmigrante La uti- lización de protocolos y guías de práctica clínica son un elemento imprescindible para garantizar la calidad de la asistencia sanitaria y disminuir la variabilidad en la práctica clínica. En la atención al paciente inmigrante, estas guías de práctica clínica han de comprender los siguientes aspectos: integrar el eje preventivo y el eje asistencial (no se puede establecer estrictamente un binomio salud-enfermedad); considerar los mecanismos de adaptación sociolaboral y de situación legal como uno de los primeros condicionantes para enfermar de la población inmigrante; establecer algoritmos diferenciales para patologías diferenciales, no para poblaciones diferenciales; describir contenidos sociosanitarios de atención al inmigrante; establecer los niveles de coordinación entre niveles asistenciales para aquellos casos en que sea necesaria una atención hospitalaria; reflejar la realidad multicultural y los condicionantes de salud-enfermedad de las distintas poblaciones inmigrantes. El punto de partida ha de ser, como en la aplicación de cualquier guía clínica, un cuidadoso respeto por las creencias, conocimientos y actitudes de los pacientes. El manejo de estas guías clínicas servirá especialmente para la actuación ante aquellas patologías diferentes que no se está acostumbrado a manejar en atención primaria.

e. Reorganización interna de los equipos de atención primaria. Los equipos de atención primaria como primer nivel de prestación de servicios sanitarios deben reformular sus estrategias y adecuar sus servicios a la nueva situación. Para ello deben de conocer la prevalencia de la población inmigrante que pertenece a su zona básica de salud y conocer su origen, cultura, hábitos de vida, hábitos dietéticos, costumbres, etc. Esto garantizará una mejor adaptación y una correcta atención. El conocimiento por parte del personal sanitario y no sanitario de la realidad cultural de la población inmigrante a la que atiende puede hacer cambiar sus actitudes frente a ellos. 
Debería implementarse una visita inicial de acogida a inmigrantes nuevos con objeto de informar sobre los recursos existentes en el centro, la forma de obtenerlos, iniciación de la historia clínica y aumentar la confianza del paciente inmigrante en el sistema sanitario. El papel de enfermería en esta visita de acogida es fundamental.

El desarrollo de un trabajo con protocolos facilitaría la conexión y comunicación con las unidades especializadas de enfermedades importadas para el asesoramiento y la solución de casos que requieran mayor nivel de complejidad. Estas unidades deben de utilizarse como recursos de apoyo a la atención primaria.

El papel de las unidades de trabajo social de atención primaria es clave para establecer una correcta coordinación con las unidades de trabajo social municipales y con otras organizaciones de inmigrantes que trabajen en la comunidad. De esta forma se realizará un correcto abordaje biopsicosocial de la población atendida.

La disponibilidad de hojas de anamnesis, órdenes de tratamiento y consejos de educación para la salud (dietas, adherencia a tratamientos, recomendaciones vacunales...) en varios idiomas y culturalmente adaptadas a las peculiaridades de las comunidades de inmigrantes predominantes en la zona básica, facilitaría el trabajo al personal sanitario y aumentaría la calidad de la atención prestada. En ocasiones, los equipos deberán adaptar los programas que ya se aplican y/o crear algunos específicos.

\section{BIBLIOGRAFIA}

1. Gámez Gámez E, Galindo JP, Cañada JL, Coll J, DíAz M, HernÁndez GonZÁlez E. La atención al inmigrante: del aluvión a la solución razonable. Documentos Sociedad Española de Medicina Familiar y Comunitaria (semFYC), 2002.

2. GiméNEZ Romero C. Dinamización comunitaria en el ámbito de la inmigración. En: Rubio MJ y Monteros S. La exclusión social. Teroría y práctica de la intervención. Madrid: CCS 2002: 99-125.

3. Díaz Olalla JM. Podrá la población inmigrante superar el impacto de nuestro nivel de salud? Aten Prim 2005; 36 (Supl 1): 38-39.
4. Documentos Técnicos Salud Pública $n^{\circ} 91$. Inmigrantes, salud y servicios sanitarios. La perspectiva de la población inmigrante. Madrid: Instituto de Salud Pública. Consejería de Sanidad y Consumo de la Comunidad de Madrid 2005.

5. Documentos Técnicos Salud Pública nº 84 . Estudio del mapa alimentario de la población inmigrante residente en la comunidad de Madrid. Madrid: Instituto de Salud Pública. Consejería de Sanidad y Consumo de la Comunidad de Madrid 2005.

6. BARBERO J. Inmigración, salud y bioética. En: Alonso A, Huerga H, Morera J. Guía de atención al inmigrante. Madrid: Ergon 2003: 4555.

7. SANZ B, TorRes AM. Situación sociolaboral y accidentes referidos por la población marroquí en un área de la comunidad de Madrid. Aten Prim 2000; 26: 314-318.

8. Carballo M, Divino JL, Zeric D. Migration and healthh in the European Union. Trop Med Int Health 1998; 3: 936-944.

9. Castillo S, Mazarrasa L, Sanz B. Mujeres inmigrantes hablando de su salud. Index Enferm 2001; 34: 9-14.

10. JANSÁ JM. Inmigración extranjera en el estado español. Consideraciones desde la salud pública. Rev Esp Salud Pública 1998;72:165168.

11. Guerrero A, Colomina J. Población inmigrante y enfermedades infecciosas. Rev Esp Salud Pública 2004; 78: 565-570.

12. GASCón J. Enfermedades infecciosas e inmigración. Enferm Infecc Microbiol Clin 2003; 21: 535-539.

13. Farias HuAnQui P, editores. Manual de atención primaria a población inmigrante. Madrid: Artur S. A., 2001.

14. Bartolemé M, Balanzó X, Roca C, Ferrer P, FERnÁndez Roure JL et al. Paludismo importado: una enfermedad emergente. Med Clin (Bar) 2002; 119: 616-619.

15. LóPEZ-VÉlez R, PÉrez-Casas C, Martín-Aresti J, Turrientes MC, García-CAMACHO A. Clinicoepidemiological study of imported malaria in travelers and inmigrants to Madrid. J Travel Med 1999; 6: 81-86.

16. Boletín epidemiológico de la Comunidad Autónoma de Madrid 2002; 8: 24-26.

17. ChIN DP, Deriemer K. Differences in contributing factors to tuberculosis incidence in USborns and foreing-born persons. Am J Respir Crit care Med 1998; 158: 1797-1803.

18. Gutierre MC, Vincent V, Aubert D, Bizet J, PieRRE-AudigiER C. Institute Pasteur. Molecular 
fingerperinting of Mycobacterium tuberculosis transmission in Paris, France and sourrounding area. J Clin Microbiol 1998; 36: 486-492.

19. IÑIgo J, Chaves F, Arce A, Palenque E. Transmisión reciente de la tuberculosis en Madrid: utilidad de técnicas moleculares. Med Clin (Bar) 2000; 115: 241-245.

20. Bates JH, Stead WW. Historia de la tuberculosis como epidemia globla. Clin Med North AM 1993; 6: 1277-1288.

21. Huerga H, López-Velez R, Navas E, Gomez-MamPASO E. Clinicaepidemiological features of inmigrants of tuberculosis living in Madrid, Spain. Eur J Clin Microbiol Infect Dis 2000; 19: 236-240.

2 2. Ruiz Manzano J. Tuberculosis e inmigración. Med Clin (Bar) 2000; 214: 257-258.

23. Alonso FJ, García MC, Lougedo MJ, Comas JM, García M, López de CASTRo F et al. Prevalencia de infección tuberculosa en las personas inmigrantes del área de Toledo. Rev Esp Salud Pública 2004; 78: 593-600.

24. Fernández MT, Díaz J, Sánchez JM, Pérez A, VADILlo J. Prevalencia de infección tuberculosa en la población de inmigrantes en Ceuta, España. Rev Esp Salud Pública 2001; 75: 551-558.

25. Rivas FPJ, NÁcher M, Corrillero J. Prevalencia de infección tuberculosa en inmigrantes magrebíes. Med Clin (Barc) 2000; 114: 245249.

26. Grupo de Trabajo del PMIT. Incidencia de la tuberculosis en España: resultados del Proyecto Multicéntrico de Investigación en Tuberculosis. Med Clin (Barc) 2000; 114: 530-537.

27. VÁzquez J, Galindo JP, LuJÁn R, GÁMEZ E, RAMOS JA, LORENTE J et al. Atención al paciente inmigrante en atención primaria. Med Fam 2000; 1: 50.

28. Seguí M. El inmigrante en la consulta del médico de cabecera. Semergen 2005; 31: 505-507.

29. Pena M. Motivos de consulta y características demográficas de una comunidad de inmigrantes "sin papeles" en el distrito de Usera-Villaverde (Madrid). Aten Prim 2001; 27: 25-28.

30. Médicos del Mundo-Comunidad Valenciana. Informe de Exclusión de médicos del Mundo, 2003.

31. Ballesteros AM, Anaya JA, CÁmara MA, López J, PAgan E, GhelichKhani M. El paciente inmigrante: ¿una realidad diferente? Semergen 2005; 31: 516-520.
32. Sánchez R, Soto M, Pérez N, Alonso FJ, De Castro C, García MC. Motivos de consulta y características sociosanitarias de la población inmigrante del área de salud de Toledo. Semergen 2005; 31: 408-412.

33. VÁzQuEZ J. Asistencia al inmigrante desde el equipo de atención primaria (aspectos organizativos, formativos y de planificación). Cuadernos de Gestión 2002; 8: 54-60.

34. Tızon JL. Migraciones y salud mental: recordatorio. Gac Sanitaria 1989; 13: 283-289.

35. EkBlad S, KoHn R, JANSSON B. Psychological and clinical aspects of immigration and mental health. En: Clinical methods in transcultural psychiatry. SO Okpaku. Washington: American Psychiatric Press, 1998.

36. Achotegui J. Inmigrantes en situación extrema. El síndrome del inmigrante con estrés crónico y múltiple. El Medico 2004; 7 mayo: 18-20.

37. GRACIA D. ¿Qué es un sistema justo de servicios de salud? Principios para la asignación de recursos escasos. Boletín de la Oficina Sanitaria Panamericana 1990; 108, n 5-6: 187-201.

38. DRANE J. Cuestiones de justicia en la prestación de servicios de salud. Boletín de la Oficina Sanitaria Panamericana 1990; 108, nº 56: 202-214.

39. De LuCAs MarTín J. Integración, inmigración, derechos humanos. En: Rubio MJ, Monteros $\mathrm{S}$ (Cord). La exclusión social: teoría y práctica de la intervención. Madrid: CCS, 2002; 7198 .

40. Martincano JL. Manual de Medicina Transcultural. $1^{\text {a }}$ ed. Madrid: IM\&C, S.A.; 2003: 75128.

41. Borrel I, CARrió F. Entrevista clínica. Manual de estrategias prácticas. Barcelona: Semfyc Ediciones 2004.

42. Ruiz Moral R. Relación clínica. Guía para aprender, enseñar e investigar. Barcelona: Semfyc Ediciones 2004.

43. Stewart M, Belle Brown J, Weston W, McWhinNey I, McWillian C, FreEman T. Pt-Centered medicine: transforming the clinical method. thousand oaks, CA: Sage Publications 1995.

44. SÁNCHEZ T. La inmigración, reto nuevo en la comunicación médico-paciente. Salud Rural 2003; 20: 39-45.

45. Sociedad Española de Medicina Familiar y Comunitaria (semFYC). Recomendaciones para la atención al paciente inmigrante en la consulta de Atención Primaria. Barcelona: semFYC 2001. Disponible en http//www. semfyc.es. 
46. Consejería de salud de Andalucía. Manual de atención sanitaria a inmigrantes. Sevilla: $2^{a}$ edición Fundación Progreso y Salud Consejería de Salud Junta de Andalucía 2004.

47. Sociedad Madrileña de Medicina Familiar y Comunitaria. Guía de Atención al inmigrante. Madrid: Ergon 2003.

48. Jansá JM, García de Olalla P. Salud e inmigración: nuevas realidades y nuevos retos. Gac Sanit 2004; 18 (Supl): 207-213.
49. Alonso E. Gestión de la diversidad cultural en atención primaria. Cuadernos de Gestión para el profesional de atención primaria 2002; 8: 49-53.

50. Alonso E. El médico de familia ante el fenómeno migratorio. Salud e inmigración. Estrategias y recursos en Cataluña. FMC 2004; 11(Supl 3): 5. 
